\title{
Some Fixed-Point Results via Mix-Type Contractive Condition
}

\author{
Özlem Acar (10 \\ Faculty of Science, Department of Mathematics, Selçuk University, 42003 Konya, Turkey \\ Correspondence should be addressed to Özlem Acar; ozlem.acar@selcuk.edu.tr \\ Received 1 February 2021; Revised 12 February 2021; Accepted 27 February 2021; Published 10 March 2021 \\ Academic Editor: Ioan Rasa \\ Copyright ( 2021 Özlem Acar. This is an open access article distributed under the Creative Commons Attribution License, which \\ permits unrestricted use, distribution, and reproduction in any medium, provided the original work is properly cited.
}

We consider a fixed-point problem for mappings involving a rational type and almost type contraction on complete metric spaces. To do this, we are using $F$-contraction and $(H, \varphi)$-contraction. We also present an example to illustrate our result.

\section{Introduction}

The beginning of metrical fixed point theory is related to Banach's Contraction Principle, presented in 1922 [1], which says that any contraction self-map on $M$ has a unique fixed point whenever $(M, d)$ is complete. Afterwards, the crucial role of the principle in existence and uniqueness problems arising in mathematics has been realized which fact directed the researchers to extend and generalize the principle in many ways (see [2-7]).

In the studies of generalizations and modifications of contractions, an interesting generalization was given by Wardowski [8] using a new concept $F$-contraction. Then, many authors gave some results using this concept in different type metric spaces. One of them is given by Jleli et al. [9] by introducing a family $\mathscr{H}$ of functions $\left.H:[0, \infty)^{3} \rightarrow 0, \infty\right)$ with the certain assumption. Also, you can find this type generalizations in [10-12].

In this paper, we consider a fixed-point problem for mappings involving a rational type contraction and almost contraction. Firstly, we recall some basic on the notions of $F$ -contraction and $(H, \phi)$-contraction.

\section{Preliminaries}

Let $\mathscr{F}$ be the family of all functions $F: \mathbb{R}^{+}=[0, \infty) \rightarrow \mathbb{R}$ satisfying the following conditions:

$(F 1) F$ is nondecreasing;

(F2) for every sequence $\left\{\alpha_{n}\right\}$ of positive numbers $\lim _{n \rightarrow+\infty} \alpha_{n}=0$ if and only if $\lim _{n \rightarrow+\infty} F\left(\alpha_{n}\right)=-\infty$; $([8])$

(F3) there exists $k \in] 0,1\left[\right.$ such that $\lim _{\alpha \rightarrow 0^{+}} \alpha^{k} F(\alpha)=0$.

Definition 1. (see [8]). Let $(M, d)$ be a metric space and $Y$ $: M \rightarrow M$ be a mapping. Given $F \in \mathscr{F}$, we say that $Y$ is $F$ -contraction, if there exists $\tau>0$ such that

$$
\mu, \gamma \in M, d(Y \mu, Y \gamma)>0 \Rightarrow \tau+F(d(Y \mu, Y \gamma)) \leq F(d(\mu, \gamma)) .
$$

Taking in (1) different functions $F \in \mathscr{F}$, one gets a variety of $F$-contractions, and some of them being already known in the literature. You can see this contractions in [8]. In addition, Wardowski concluded that every $F$-contraction $Y$ is a contractive mapping, i.e.,

$$
d(Y \mu, Y \gamma)<d(\mu, \gamma) \text {, forall } \mu, \gamma \in M, Y \mu \neq Y \gamma .
$$

Thus, every F-contraction is a continuous mapping.

Theorem 2. (see [8]). Let $(M, d)$ be a complete metric space (C.M.S) and let $Y: M \rightarrow M$ be an F-contraction. Then, $Y$ has a unique fixed point in $M$.

In [9], Jleli et al. introduced a family $\mathscr{H}$ of functions $H$ $:[0,+\infty)^{3} \rightarrow 0,+\infty$ satisfying the following conditions:

(H1) $\max \{\alpha, \beta\} \leq H(\alpha, \beta, \gamma)$ for all $\alpha, \beta, \gamma \in 0,+\infty$;

(H2) $H(0,0,0)=0$;

(H3) $H$ is continuous.

Some examples of functions belonging to $\mathscr{H}$ are given as follows: 
(i) $H(\alpha, \beta, \gamma)=\alpha+\beta+\gamma$ for all $\alpha, \beta, \gamma \in 0,+\infty$

(ii) $H(\alpha, \beta, \gamma)=\max \{\alpha, \beta\}+\gamma$ for all $\alpha, \beta, \gamma \in 0,+\infty$

(iii) $H(\alpha, \beta, \gamma)=\alpha+\beta+\alpha \beta+\gamma$ for all $\alpha, \beta, \gamma \in 0,+\infty$

Using a function $H \in \mathscr{H}$, the authors of [9] introduced the following notion of $(H, \phi)$-contraction.

Definition 3. (see [9]). Let $(M, d)$ be a metric space, $\phi: M$ $\rightarrow 0,+\infty$ be a given function, and $H \in \mathscr{H}$. Then, $Y: M \rightarrow$ $M$ is called a $(H, \phi)$-contraction with respect to the metric $d$ if and only if

$$
\begin{aligned}
& H(d(Y \mu, Y \gamma), \phi(Y \mu), \phi(Y \gamma)) \\
& \quad \leq k H(d(\mu, \gamma), \phi(\mu), \phi(\gamma)) \text { for all } \mu, \gamma \in M,
\end{aligned}
$$

for some constant $k \in] 0,1[$.

Now, we set

$$
\begin{gathered}
Z_{\phi}:=\{\mu \in M: \phi(\mu)=0\}, \\
F_{Y}:=\{\mu \in M: Y \mu=\mu\} .
\end{gathered}
$$

Furthermore, we say that $Y$ is a $\phi$-Picard operator if and only if the following condition holds

$$
F_{Y} \cap Z_{\phi}=\{\varsigma\} \text { and } Y^{n} \mu \rightarrow \varsigma \text {, as } n \rightarrow+\infty \text {,for each } \mu \in M \text {. }
$$

Theorem 4. (see [9]). Let $(M, d)$ be a C.M.S, $\phi: M \rightarrow 0,+\infty$ be a given function and $H \in \mathscr{H}$. Suppose that the following conditions hold

(A1) $\phi$ is lower semicontinuous (l.s.c.);

(A2) $Y: M \rightarrow M$ is a $(H, \phi)$-contraction with respect to the metric $d$.

Then,

$$
F_{Y} \subset Z_{\phi}
$$

(i) $Y$ is a $\phi$-Picard operator

(ii) For all $\mu \in M$ and for all $n \in \mathbb{N}$, we have

$$
d\left(Y^{n} \mu, \varsigma\right) \leq \frac{k^{n}}{1-k} H(d(Y \mu, \mu), \phi(Y \mu), \phi(\mu)),
$$

where $\{\varsigma\}=F_{Y} \cap Z_{\phi}=F_{Y}$.

Recently, Vetro ([13]) generalized Theorem 4 by using $F$ -H-contraction.

Definition 5. (see [13]). Let $(M, d)$ be a metric space and let $Y: M \rightarrow M$ be a mapping. The mapping $Y$ is called an $F$ $H$-contraction if there exists $F \in \mathscr{F}, H \in \mathscr{H}$, a real number, $\tau>0$ and $\phi: M \rightarrow 0,+\infty)$ s.t.
$\tau+F(H(d(Y \mu, Y \gamma), \phi(Y \mu), \phi(Y \gamma))) \leq F(H(d(\mu, \gamma), \phi(\mu), \phi(\gamma)))$,

for all $\mu, \gamma \in M$ with $H(d(Y \mu, Y \gamma), \phi(Y \mu), \phi(Y \gamma))>0$.

We remark that every $F$-contraction is an $F$-H-contraction such that $H \in \mathscr{H}$ defined by $H(x, y, z)=x+y+z$ for all $x, y, z \in 0,+\infty$ and $\phi: M \rightarrow 0,+\infty$ defined by $\phi(\mu)=0$ for all $\mu \in M$.

Lemma 6. (see [13]). Let $(M, d)$ be a metric space and let $Y$ $: M \rightarrow M$ be an $F-H$-contraction with respect to the functions $F \in \mathscr{F}, H \in \mathscr{H}, \phi: M \rightarrow 0,+\infty$, and the real number $\tau>0$. If $\left\{\mu_{n}\right\}$ is a sequence of Picard starting at $\mu_{0} \in M$, then

$$
\lim _{n \rightarrow+\infty} H\left(d\left(\mu_{n-1}, \mu_{n}\right), \phi\left(\mu_{n-1}\right), \phi\left(\mu_{n}\right)\right)=0,
$$

and hence

$$
\lim _{n \rightarrow+\infty} d\left(\mu_{n-1}, \mu_{n}\right)=0 \text { and } \lim _{n \rightarrow+\infty} \phi\left(\mu_{n}\right)=0
$$

Theorem 7. (see [13]). Let $(M, d)$ be a C.M.S and $Y: M \rightarrow$ $M$ be an $F-H$-contraction with respect to the functions $F \in$ $\mathscr{F}, H \in \mathscr{H}$, the real number $\tau>0$, and a l.s.c. function $\phi: M$ $\rightarrow 0,+\infty$ such that (8) holds; that is,

$\tau+F(H(d(Y \mu, Y \gamma), \phi(Y \mu), \phi(Y \gamma))) \leq F(H(d(\mu, \gamma), \phi(\mu), \phi(\gamma)))$,

for all $\mu, \gamma \in M$ with $H(d(Y \mu, Y \gamma), \phi(Y \mu), \phi(Y \gamma))>0$. Then, $Y$ has a unique fixed point $\varsigma$ such that $\phi(\varsigma)=0$.

Theorem 8. (see [13]). Let $(M, d)$ be a C.M.S and let $Y: M$ $\rightarrow M$ be a mapping. Assume that there exists a continuous function $F$ that satisfies the conditions $\left(F_{1}\right)$ and $\left(F_{2}\right)$, a function $H \in \mathscr{H}$, a real number $\tau>0$, and a l.s.c. function $\phi: M$ $\rightarrow 0,+\infty$ such that (8) holds; that is,

$\tau+F(H(d(Y \mu, Y \gamma), \phi(Y \mu), \phi(Y \gamma))) \leq F(H(d(\mu, \gamma), \phi(\mu), \phi(\gamma)))$,

for all $\mu, \gamma \in M$ with $H(d(Y \mu, Y \gamma), \phi(Y \mu), \phi(Y \gamma))>0$. Then, $Y$ has a unique fixed point $\varsigma$ such that $\phi(\varsigma)=0$.

\section{Main Results}

We first introduce the rational type $F-H$-contraction.

Definition 9. Let $(M, d)$ be a metric space and $Y: M \rightarrow M$ be a mapping. $Y$ is called a rational type $F-H$-contraction if there exists $F \in \mathscr{F}, H \in \mathscr{H}$, a real number $\tau>0$, and $\phi: M$ $\rightarrow 0,+\infty$ s.t.

$\tau+F(H(d(Y \mu, Y \gamma), \phi(Y \mu), \phi(Y \gamma))) \leq F(H(M(\mu, \gamma), \phi(\mu), \phi(\gamma)))$, 
for all $\mu, \gamma \in M$ with $H(d(Y \mu, Y \gamma), \phi(Y \mu), \phi(Y \gamma))>0$ where

$$
M(\mu, \gamma)=\max \left\{d(\mu, \gamma), \frac{d(\mu, Y \mu)[1+d(\gamma, Y \gamma))]}{1+d(Y \mu, Y \gamma)}\right\} .
$$

Lemma 10. Let $(M, d)$ be a metric space and $Y: M \rightarrow M$ be a rational type $F-H$-contraction with respect to the functions $F \in \mathscr{F}, H \in \mathscr{H}, \phi: M \rightarrow 0,+\infty$, and the real number $\tau>0$. If $\left\{\mu_{n}\right\}$ is a sequence of Picard starting at $\mu_{0} \in M$, then

$$
\lim _{n \rightarrow+\infty} H\left(d\left(\mu_{n-1}, \mu_{n}\right), \phi\left(\mu_{n-1}\right), \phi\left(\mu_{n}\right)\right)=0,
$$

and hence

$$
\lim _{n \rightarrow+\infty} d\left(\mu_{n-1}, \mu_{n}\right)=0 \text { and } \lim _{n \rightarrow+\infty} \phi\left(\mu_{n}\right)=0 .
$$

Proof. By replacing the contradiction in [[13], (29)] with contradiction (13) and following the proof of [[13], Lemma 1], we immediately have the desired result.

Theorem 11. Let $(M, d)$ be a C.M.S and let $Y: M \rightarrow M$ be an rational type $F-H$-contraction with respect to the functions $F \in \mathscr{F}, H \in \mathscr{H}$, the real number $\tau>0$, and a l.s.c. function $\phi$ $: M \rightarrow 0,+\infty$ such that (13) holds for all $\mu, \gamma \in M$ with $H(d($ $Y \mu, Y \gamma), \phi(Y \mu), \phi(Y \gamma))>0$. Then, $Y$ has a unique fixed point $\varsigma$ such that $\phi(\varsigma)=0$.

Proof. First, we shall proof the uniqueness. Arguing by contradiction, we assume that there exist $\varsigma, w \in M$ such that $\varsigma$ $=Y \varsigma, w=Y w$, and $\varsigma \neq w$. The hypothesis $\varsigma \neq w$ ensures, by the property $\left(H_{1}\right)$ of the function $H$, that

$$
H(d(Y \varsigma, Y w), \phi(Y \varsigma), \phi(Y w)) \geq d(Y \varsigma, Y w)=d(\varsigma, w)>0 .
$$

Using (13) with $\mu=\varsigma$ and $\gamma=w$, we obtain

$$
\begin{aligned}
\tau & +F(H(d(Y \varsigma, Y w), \phi(Y \varsigma), \phi(Y w))) \\
& =\tau+F(H(d(\varsigma, w), \phi(\varsigma), \phi(w))) \\
& \leq F(H(M(\varsigma, w), \phi(\varsigma), \phi(w))) \\
& \leq F\left(H\left(\max \left\{d(\varsigma, w), \frac{d(\varsigma, Y \varsigma)[1+d(w, Y w))]}{1+d(Y \varsigma, Y w)}\right\}, \phi(\varsigma), \phi(w)\right)\right) \\
& \leq F\left(H\left(\max \left\{d(\varsigma, w), \frac{d(\varsigma, \varsigma)[1+d(w, w))]}{1+d(\varsigma, w)}\right\}, \phi(\varsigma), \phi(w)\right)\right) \\
& \leq F(H(d(\varsigma, w), \phi(\varsigma), \phi(w))),
\end{aligned}
$$

which is a contradiction. So, we have $w=\varsigma$, and the fixed point is unique.

Now, we can show the existence of a fixed point. Take a point $\mu_{0} \in M$ and create the $\left\{\mu_{n}\right\}$ sequence starting at $\mu_{0}$. We emphasize that if $\mu_{k-1}=\mu_{k}$ for some $k \in \mathbb{N}$, then $\varsigma=$ $\mu_{k-1}=\mu_{k}=Y \mu_{k-1}=Y \varsigma$; that is, $\varsigma$ is a fixed point of $Y$ such that $\phi(\varsigma)=0$. In fact, by Lemma $10, H\left(d\left(\mu_{k-1}, \mu_{k}\right), \phi\left(\mu_{k-1}\right)\right.$, $\left.\phi\left(\mu_{k}\right)\right)=0$ and by the property $\left(H_{1}\right)$ of the function $H$, we have $\phi(\varsigma)=0$. So, we can suppose that $\mu_{n-1} \neq \mu_{n}$ for every $n$ $\in \mathbb{N}$.

In this step, we show that $\left\{\mu_{n}\right\}$ is a Cauchy. By Lemma 10 , we say that

$$
0<h_{n-1}=H\left(d\left(\mu_{n-1}, \mu_{n}\right), \phi\left(\mu_{n-1}\right), \phi\left(\mu_{n}\right)\right) \rightarrow 0 \text { as } n \rightarrow+\infty \text {. }
$$

There exists $k \in] 0,1\left[\right.$ such that $h_{n}^{k} F\left(h_{n}\right) \rightarrow 0$ as $n \rightarrow+\infty$ by he property $\left(F_{3}\right)$ of $F$. Using (13) with $\mu=\mu_{n-1}$ and $\gamma=$ $\mu_{n}$, we get

$$
\begin{aligned}
F( & \left.H\left(d\left(\mu_{n}, \mu_{n+1}\right), \phi\left(\mu_{n}\right), \phi\left(\mu_{n+1}\right)\right)\right) \\
\leq & F\left(H\left(M\left(\mu_{n-1}, \mu_{n}\right), \phi\left(\mu_{n-1}\right), \phi\left(\mu_{n}\right)\right)\right)-\tau \\
\leq & F\left(H \left(\operatorname { m a x } \left\{d\left(\mu_{n-1}, \mu_{n}\right),\right.\right.\right. \\
& \left.\left.\left.\quad \cdot \frac{\left.d\left(\mu_{n-1}, Y \mu_{n-1}\right)\left[1+d\left(\mu_{n}, Y \mu_{n}\right)\right)\right]}{1+d\left(Y \mu_{n-1}, Y \mu_{n}\right)}\right\}, \phi\left(\mu_{n-1}\right), \phi\left(\mu_{n}\right)\right)\right)-\tau \\
\leq & F\left(H\left(d\left(\mu_{n-1}, \mu_{n}\right), \phi\left(\mu_{n-1}\right), \phi\left(\mu_{n}\right)\right)\right)-\tau \\
\leq & F\left(H\left(d\left(\mu_{0}, \mu_{1}\right), \phi\left(\mu_{0}\right), \phi\left(\mu_{1}\right)\right)\right)-n \tau,
\end{aligned}
$$

for all $n \in \mathbb{N}$; that is,

$$
F\left(h_{n}\right) \leq F\left(h_{n-1}\right)-\tau \leq \cdots \leq F\left(h_{0}\right)-n \tau \text { for all } n \in \mathbb{N} .
$$

From

$$
0=\lim _{n \rightarrow+\infty} h_{n}^{k} F\left(h_{n}\right) \leq \lim _{n \rightarrow+\infty} h_{n}^{k}\left(F\left(h_{0}\right)-n \tau\right) \leq 0,
$$

we deduce that

$$
\lim _{n \rightarrow+\infty} h_{n}^{k} n=0
$$

This provides that $\sum_{n=1}^{+\infty} h_{n}$ is convergent. By the property $\left(H_{1}\right)$ of the function $H$, also, the series $\sum_{n=1}^{+\infty} d\left(\mu_{n}, \mu_{n+1}\right)$ is convergent and hence $\left\{\mu_{n}\right\}$ is a Cauchy sequence. Now, since $(M, d)$ is complete, there exists $\varsigma \in M$ such that

$$
\lim _{n \rightarrow+\infty} \mu_{n}=\varsigma
$$

By (13), taking into account that $\phi$ is a l.s.c. function, we have

$$
0 \leq \phi(\varsigma) \leq \liminf _{n \rightarrow+\infty} \phi\left(\mu_{n}\right)=0
$$

that is, $\phi(\varsigma)=0$. Now, show that $\varsigma$ is a fixed point. If there exists a subsequence $\left\{\mu_{n_{k}}\right\}$ of $\left\{\mu_{n}\right\}$ such that $\mu_{n_{k}}=\varsigma$ or $Y$ $\mu_{n_{k}}=Y_{\varsigma}$, for all $k \in \mathbb{N}$, then $\varsigma$ is a fixed point. Otherwise, we can assume that $\mu_{n} \neq \varsigma$ and $Y \mu_{n} \neq Y \varsigma$ for all $n \in \mathbb{N}$. So, using (13) with $\mu=\mu_{n}$ and $\gamma=\varsigma$, we deduce that

$$
\begin{gathered}
\tau+F\left(H\left(d\left(Y \mu_{n}, Y \varsigma\right), \phi\left(Y \mu_{n}\right), \phi(Y \varsigma)\right)\right) \\
\leq F\left(H\left(M\left(\mu_{n}, \varsigma\right), \phi\left(\mu_{n}\right), \phi(\varsigma)\right)\right) .
\end{gathered}
$$


Since $\tau>0$, we obtain

$$
\begin{aligned}
& H\left(d\left(Y \mu_{n}, Y \varsigma\right), \phi\left(Y \mu_{n}\right), \phi(Y \varsigma)\right) \\
& \quad<H\left(M\left(\mu_{n}, \varsigma\right), \phi\left(\mu_{n}\right), \phi(\varsigma)\right) \text { for all } n \in \mathbb{N},
\end{aligned}
$$

and so

$$
\begin{aligned}
d(\varsigma, Y \varsigma) \leq & d\left(\varsigma, \mu_{n+1}\right)+d\left(Y \mu_{n}, Y \varsigma\right) \\
\leq & d\left(\varsigma, \mu_{n+1}\right)+H\left(d\left(Y \mu_{n}, Y \varsigma\right), \phi\left(Y \mu_{n}\right), \phi(Y \varsigma)\right) \\
< & d\left(\varsigma, \mu_{n+1}\right)+H\left(M\left(\mu_{n}, \varsigma\right), \phi\left(\mu_{n}\right), \phi(\varsigma)\right) \\
< & d\left(\varsigma, \mu_{n+1}\right)+H\left(\operatorname { m a x } \left\{d\left(\mu_{n}, \varsigma\right),\right.\right. \\
& \left.\left.\cdot \frac{\left.d\left(\mu_{n}, Y \mu_{n}\right)[1+d(\varsigma, Y \varsigma))\right]}{1+d\left(Y \mu_{n}, Y \varsigma\right)}\right\}, \phi\left(\mu_{n}\right), \phi(\varsigma)\right) \\
\leq & d\left(\varsigma, \mu_{n+1}\right)+H\left(\operatorname { m a x } \left\{d\left(\mu_{n}, \varsigma\right),\right.\right. \\
& \left.\left.\cdot \frac{\left.d\left(\mu_{n}, \mu_{n+1}\right)[1+d(\varsigma, Y \varsigma))\right]}{1+d\left(\mu_{n+1}, Y \varsigma\right)}\right\}, \phi\left(\mu_{n}\right), \phi(\varsigma)\right),
\end{aligned}
$$

for all $n \in \mathbb{N}$.

Finally, letting $n \rightarrow+\infty$ in the above calculations and using that $H$ is continuous in $(0,0,0)$, we deduce that $d(\varsigma$, $Y \varsigma) \leq H(0,0,0)=0$; that is, $\varsigma=Y \varsigma$.

Imposing that $F$ is a continuous function and relaxing the hypothesis $\left(F_{3}\right)$, we can give $t$ Theorem 12 .

Theorem 12. Let $(M, d)$ be $a$ C.M.S and $Y: M \rightarrow M$ be $a$ mapping. Assume that there exists a continuous function $F$ that satisfies the conditions $\left(F_{1}\right)$ and $\left(F_{2}\right)$, a function $H \in \mathscr{H}$, a real number $\tau>0$, and a l.s.c. function $\phi: M \rightarrow 0,+\infty$ s.t.

$$
\begin{gathered}
\tau+F(H(d(Y \mu, Y \gamma), \phi(Y \mu), \phi(Y \gamma))) \\
\leq F(H(M(\mu, \gamma), \phi(\mu), \phi(\gamma))),
\end{gathered}
$$

for all $\mu, \gamma \in M$ with $H(d(Y \mu, Y \gamma), \phi(Y \mu), \phi(Y \gamma))>0$. Then, $Y$ has a unique fixed point $\varsigma$ such that $\phi(\varsigma)=0$.

Proof. Following the similar arguments as in the proof of Theorem 11, we obtain easily the uniqueness of the fixed point. The existence of a fixed point, we take a point $\mu_{0} \in M$ and create the $\left\{\mu_{n}\right\}$ sequence starting at $\mu_{0}$. Clearly, if $\mu_{k-1}$ $=\mu_{k}$ for some $k \in \mathbb{N}$, then $\varsigma=\mu_{k-1}=\mu_{k}=Y \mu_{k-1}=Y \varsigma$; that is, $\varsigma$ is a fixed point of $Y$ such that $\phi(\varsigma)=0$ (see the proof of Theorem 11), and so we have already done.

So, we can suppose that $\mu_{n-1} \neq \mu_{n}$ for every $n \in \mathbb{N}$. Now, showing that $\left\{\mu_{n}\right\}$ is a Cauchy. Let us admit the opposite. Then, there exists a positive real number $\varepsilon$ and two sequences $\left\{m_{k}\right\}$ and $\left\{n_{k}\right\}$ such that

$n_{k}>m_{k} \geq k$ and $d\left(\mu_{m_{k}}, \mu_{n_{k}}\right) \geq \varepsilon>d\left(\mu_{m_{k}}, \mu_{n_{k}-1}\right)$ for all $k \in \mathbb{N}$.

By Lemma 10 , we say that $d\left(\mu_{n-1}, \mu_{n}\right) \rightarrow 0, \phi\left(\mu_{n}\right) \rightarrow 0$, as $n \rightarrow+\infty$. This implies

$$
\lim _{k \rightarrow+\infty} d\left(\mu_{m_{k}}, \mu_{n_{k}}\right)=\lim _{k \rightarrow+\infty} d\left(\mu_{m_{k}-1}, \mu_{n_{k}-1}\right)=\varepsilon
$$

Now, the hypothesis that $d\left(\mu_{m_{k}}, \mu_{n_{k}}\right)>\varepsilon$ ensures that

$$
H\left(d\left(\mu_{m_{k}}, \mu_{n_{k}}\right), \phi\left(\mu_{m_{k}}\right), \phi\left(\mu_{n_{k}}\right)\right)>0 \text { for all } k \in \mathbb{N} \text {. }
$$

Using the continuity of $H$, we have

$$
\begin{aligned}
& \lim _{k \rightarrow+\infty} H\left(d\left(\mu_{m_{k}-1}, \mu_{n_{k}-1}\right), \phi\left(\mu_{m_{k}-1}\right), \phi\left(\mu_{n_{k}-1}\right)\right) \\
& \quad=\lim _{k \rightarrow+\infty} H\left(d\left(\mu_{m_{k}}, \mu_{n_{k}}\right), \phi\left(\mu_{m_{k}}\right), \phi\left(\mu_{n_{k}}\right)\right) \\
& \quad=H(\varepsilon, 0,0)>0 .
\end{aligned}
$$

Using again (29), with $\mu=\mu_{m_{k}-1}$ and $\gamma=\mu_{n_{k}-1}$, we get

$$
\begin{aligned}
& \tau+F\left(H\left(d\left(\mu_{m_{k}}, \mu_{n_{k}}\right), \phi\left(\mu_{m_{k}}\right), \phi\left(\mu_{n_{k}}\right)\right)\right) \\
& \leq F\left(H\left(M\left(\mu_{m_{k}-1}, \mu_{n_{k}-1}\right), \phi\left(\mu_{m_{k}-1}\right), \phi\left(\mu_{n_{k}-1}\right)\right)\right) \\
& \leq F\left(H\left(\max \left\{\frac{\left.d\left(\mu_{m_{k}-1}, Y \mu_{m_{k}-1}\right)\left[1+d\left(\mu_{n_{k}-1}, Y \mu_{n_{k}-1}\right)\right)\right]}{1+d\left(Y \mu_{m_{k}-1}, Y \mu_{n_{k}-1}\right)}\right\}, \phi\left(\mu_{m_{k}-1}\right), \phi\left(\mu_{n_{k}-1}\right)\right)\right) \\
& \leq F\left(H\left(\max \left\{\frac{\left.d\left(\mu_{m_{k}-1}, \mu_{m_{k}}\right)\left[1+d\left(\mu_{n_{k}-1}, \mu_{n_{k}}\right)\right)\right]}{1+d\left(\mu_{m_{k}}, \mu_{n_{k}}\right)}\right\}, \phi\left(\mu_{m_{k}-1}\right), \phi\left(\mu_{n_{k}-1}\right)\right),\right.
\end{aligned}
$$

for all $k \in \mathbb{N}$. Letting $k \rightarrow+\infty$ in the previous inequality, since the function $F$ is continuous, we get

$$
\tau+F(H(\varepsilon, 0,0))) \leq F(H(\varepsilon, 0,0)))
$$

which leads to contradiction. It follows that $\left\{\mu_{n}\right\}$ is a Cauchy sequence.

Now, since $(M, d)$ is complete, there exists some $\varsigma \in M$ such that

$$
\lim _{n \rightarrow+\infty} \mu_{n}=\varsigma
$$

By (29), using lower semicontinuity of $\phi$, we get

$$
0 \leq \phi(\varsigma) \leq \liminf _{n \rightarrow+\infty} \phi\left(\mu_{n}\right)=0
$$

that is, $\phi(\varsigma)=0$. Now, show that $\varsigma$ is a fixed point of $Y$. Clearly, $\varsigma$ is a fixed point of $Y$ if there exists a subsequence $\left\{\mu_{n_{k}}\right\}$ of $\left\{\mu_{n}\right\}$ such that $\mu_{n_{k}}=\varsigma$ or $Y \mu_{n_{k}}=Y_{\varsigma}$, for all $k \in \mathbb{N}$. Otherwise, we can assume that $\mu_{n} \neq \varsigma$ and $Y \mu_{n} \neq Y \varsigma$ for all $n \in \mathbb{N}$. Then, the property $\left(H_{1}\right)$ of the function $H$ ensures that $H\left(d\left(Y \mu_{n}, Y \varsigma\right), \phi\left(Y \mu_{n}\right), \phi(Y \varsigma)\right)>0$ for all $n \in \mathbb{N}$. So, using (29) with $\mu=\mu_{n}$ and $\gamma=\varsigma$, we deduce that 


$$
\begin{aligned}
\tau+ & F\left(H\left(d\left(Y \mu_{n}, Y \varsigma\right), \phi\left(Y \mu_{n}\right), \phi(Y \varsigma)\right)\right) \\
\leq & F\left(H\left(M\left(\mu_{n}, \varsigma\right), \phi\left(\mu_{n}\right), \phi(\varsigma)\right)\right) \\
\leq & F\left(H \left(\operatorname { m a x } \left\{d\left(\mu_{n}, \varsigma\right),\right.\right.\right. \\
& \left.\left.\left.\cdot \frac{\left.d\left(\mu_{n}, Y \mu_{n}\right)[1+d(\varsigma, Y \varsigma))\right]}{1+d\left(Y \mu_{n}, Y \varsigma\right)}\right\}, \phi\left(\mu_{n}\right), \phi(\varsigma)\right)\right) \text { for all } n \in \mathbb{N} .
\end{aligned}
$$

Since $\tau>0$, we conclude that

$$
\begin{aligned}
H( & \left.d\left(Y \mu_{n}, Y \varsigma\right), \phi\left(Y \mu_{n}\right), \phi(Y \varsigma)\right) \\
< & F\left(H \left(\operatorname { m a x } \left\{d\left(\mu_{n}, \varsigma\right)\right.\right.\right. \\
& \left.\left.\left.\cdot \frac{\left.d\left(\mu_{n}, Y \mu_{n}\right)[1+d(\varsigma, Y \varsigma))\right]}{1+d\left(Y \mu_{n}, Y \varsigma\right)}\right\}, \phi\left(\mu_{n}\right), \phi(\varsigma)\right)\right) \text { for all } n \in \mathbb{N}
\end{aligned}
$$

and so

$$
\begin{aligned}
d(\varsigma, Y \varsigma) \leq & d\left(\varsigma, \mu_{n+1}\right)+d\left(Y \mu_{n}, Y \varsigma\right) \\
\leq & d\left(\varsigma, \mu_{n+1}\right)+H\left(d\left(Y \mu_{n}, Y \varsigma\right), \phi\left(Y \mu_{n}\right), \phi(Y \varsigma)\right) \\
< & d\left(\varsigma, \mu_{n+1}\right)+H\left(\operatorname { m a x } \left\{d\left(\mu_{n}, \varsigma\right),\right.\right. \\
& \left.\left.\frac{\left.d\left(\mu_{n}, Y \mu_{n}\right)[1+d(\varsigma, Y \varsigma))\right]}{1+d\left(Y \mu_{n}, Y \varsigma\right)}\right\}, \phi\left(\mu_{n}\right), \phi(\varsigma)\right) \\
= & d\left(\varsigma, \mu_{n+1}\right)+H\left(\operatorname { m a x } \left\{d\left(\mu_{n}, \varsigma\right),\right.\right. \\
& \left.\left.\quad \frac{\left.d\left(\mu_{n}, \mu_{n+1}\right)[1+d(\varsigma, Y \varsigma))\right]}{1+d\left(\mu_{n+1}, Y \varsigma\right)}\right\}, \phi\left(\mu_{n}\right), \phi(\varsigma)\right),
\end{aligned}
$$

for all $n \in \mathbb{N}$. Finally, letting $n \rightarrow+\infty$ and using that $H$ is continuous in $(0,0,0)$, we deduce that $d(\varsigma, Y \varsigma) \leq H(0,0,0)$ $=0$; that is, $\varsigma=Y \varsigma$.

Definition 13. Let $(M, d)$ be a metric space and let $Y: M \rightarrow$ $M$ be a mapping. The mapping $Y$ is called almost $F-H$ -contraction if there exists a function $F \in \mathscr{F}, H \in \mathscr{H}$, a real number $\tau>0$, and $L \geq 0$ and a l.s.c. function $\phi: M \rightarrow 0,+\infty$ such that

$$
\begin{aligned}
\tau+ & F(H(d(Y \mu, Y \gamma), \phi(Y \mu), \phi(Y \gamma))) \\
& \leq F(H(d(\mu, \gamma)+L d(\gamma, Y \mu), \phi(\mu), \phi(\gamma))),
\end{aligned}
$$

for all $\mu, \gamma \in M$ with $H(d(Y \mu, Y \gamma), \phi(Y \mu), \phi(Y \gamma))>0$.

Theorem 14. Let $(M, d)$ be a C.M.S and let $Y: M \rightarrow M$ be an almost $F$ - $H$-contraction with respect to the functions $F \in \mathscr{F}$, $H \in \mathscr{H}$, the real number $\tau>0$, and $L \geq 0$ and a l.s.c. function $\phi: M \rightarrow 0,+\infty$ s.t.

$$
\begin{aligned}
\tau+ & F(H(d(Y \mu, Y \gamma), \phi(Y \mu), \phi(Y \gamma))) \\
& \leq F(H(d(\mu, \gamma)+L d(\gamma, Y \mu), \phi(\mu), \phi(\gamma))),
\end{aligned}
$$

for all $\mu, \gamma \in M$ with $H(d(Y \mu, Y \gamma), \phi(Y \mu), \phi(Y \gamma))>0$. Then, $Y$ has a fixed point $\varsigma$ such that $\phi(\varsigma)=0$.
Proof. The existence of a fixed point we take a point $\mu_{0} \in M$ and create the $\left\{\mu_{n}\right\}$ sequence starting at $\mu_{0}$. We stress that if $\mu_{k-1}=\mu_{k}$ for some $k \in \mathbb{N}$, then $\varsigma=\mu_{k-1}=\mu_{k}=Y \mu_{k-1}=Y \varsigma$; that is, $\varsigma$ is a fixed point of $Y$ such that $\phi(\varsigma)=0$. In fact, by Lemma $10, H\left(d\left(\mu_{k-1}, \mu_{k}\right), \phi\left(\mu_{k-1}\right), \phi\left(\mu_{k}\right)\right)=0$ and by the property $\left(H_{1}\right)$ of the function $H$, we have $\phi(\varsigma)=0$. So, we can suppose that $\mu_{n-1} \neq \mu_{n}$ for every $n \in \mathbb{N}$.

Now, showing that $\left\{\mu_{n}\right\}$ is a Cauchy. By Lemma 10, we say that

$$
0<h_{n-1}=H\left(d\left(\mu_{n-1}, \mu_{n}\right), \phi\left(\mu_{n-1}\right), \phi\left(\mu_{n}\right)\right) \rightarrow 0 \text { as } n \rightarrow+\infty .
$$

The property $\left(F_{3}\right)$ of the function $F$ ensures that there exists $k \in] 0,1\left[\right.$ such that $h_{n}^{k} F\left(h_{n}\right) \rightarrow 0$ as $n \rightarrow+\infty$. Using (42), with $\mu=\mu_{n-1}$ and $\gamma=\mu_{n}$, we get

$$
\begin{aligned}
F( & \left.H\left(d\left(\mu_{n}, \mu_{n+1}\right), \phi\left(\mu_{n}\right), \phi\left(\mu_{n+1}\right)\right)\right) \\
\quad & \leq F\left(H\left(d\left(\mu_{n-1}, \mu_{n}\right)+L d\left(\mu_{n}, Y \mu_{n-1}\right), \phi\left(\mu_{n-1}\right), \phi\left(\mu_{n}\right)\right)\right)-\tau \\
& \leq F\left(H\left(d\left(\mu_{n-1}, \mu_{n}\right), \phi\left(\mu_{0}\right), \phi\left(\mu_{1}\right)\right)\right)-\tau \\
& \leq F\left(H\left(d\left(\mu_{0}, \mu_{1}\right), \phi\left(\mu_{0}\right), \phi\left(\mu_{1}\right)\right)\right)-n \tau,
\end{aligned}
$$

for $\forall n \in \mathbb{N}$; that is,

$$
F\left(h_{n}\right) \leq F\left(h_{n-1}\right)-\tau \leq \cdots \leq F\left(h_{0}\right)-n \tau \text { for all } n \in \mathbb{N} .
$$

From

$$
0=\lim _{n \rightarrow+\infty} h_{n}^{k} F\left(h_{n}\right) \leq \lim _{n \rightarrow+\infty} h_{n}^{k}\left(F\left(h_{0}\right)-n \tau\right) \leq 0,
$$

we deduce that

$$
\lim _{n \rightarrow+\infty} h_{n}^{k} n=0
$$

This ensures that the series $\sum_{n=1}^{+\infty} h_{n}$ is convergent. By the property $\left(H_{1}\right)$ of the function $H$, also, the series $\sum_{n=1}^{+\infty} d\left(\mu_{n}\right.$, $\left.\mu_{n+1}\right)$ is convergent, and hence $\left\{\mu_{n}\right\}$ is a Cauchy sequence. Now, since $(M, d)$ is complete, there exists some $\varsigma \in M$ such that

$$
\lim _{n \rightarrow+\infty} \mu_{n}=\varsigma
$$

By (42), using lower semicontinuity of $\phi$, we get

$$
0 \leq \phi(\varsigma) \leq \liminf _{n \rightarrow+\infty} \phi\left(\mu_{n}\right)=0 ;
$$

that is, $\phi(\varsigma)=0$. We assert that $\varsigma$ is a fixed point of $Y$. Clearly, $\zeta$ is a fixed point of $Y$ if there exists a subsequence $\left\{\mu_{n_{k}}\right\}$ of $\left\{\mu_{n}\right\}$ such that $\mu_{n_{k}}=\varsigma$ or $Y \mu_{n_{k}}=Y \varsigma$, for all $k \in \mathbb{N}$. Otherwise, we can assume that $\mu_{n} \neq \varsigma$ and $Y \mu_{n} \neq Y \varsigma$ for all $n \in \mathbb{N}$. So, using (42) with $\mu=\mu_{n}$ and $\gamma=\varsigma$, we deduce that 


$$
\begin{aligned}
\tau+ & F\left(H\left(d\left(Y \mu_{n}, Y \varsigma\right), \phi\left(Y \mu_{n}\right), \phi(Y \varsigma)\right)\right) \\
& \leq F\left(H\left(d\left(\mu_{n}, \varsigma\right)+L d\left(\varsigma, Y \mu_{n}\right), \phi\left(\mu_{n}\right), \phi(\varsigma)\right)\right) .
\end{aligned}
$$

Since $\tau>0$, this inequality leads to

$$
\begin{aligned}
& H\left(d\left(Y \mu_{n}, Y \varsigma\right), \phi\left(Y \mu_{n}\right), \phi(Y \varsigma)\right) \\
& \quad<H\left(d\left(\mu_{n}, \varsigma\right)+L d\left(\varsigma, Y \mu_{n}\right), \phi\left(\mu_{n}\right), \phi(\varsigma)\right) \text { for all } n \in \mathbb{N}
\end{aligned}
$$

and so

$$
\begin{aligned}
d(\varsigma, Y \varsigma) & \leq d\left(\varsigma, \mu_{n+1}\right)+d\left(Y \mu_{n}, Y \varsigma\right) \\
& \leq d\left(\varsigma, \mu_{n+1}\right)+H\left(d\left(Y \mu_{n}, Y \varsigma\right), \phi\left(Y \mu_{n}\right), \phi(Y \varsigma)\right) \\
& <d\left(\varsigma, \mu_{n+1}\right)+H\left(d\left(\mu_{n}, \varsigma\right)+L d\left(\varsigma, Y \mu_{n}\right), \phi\left(\mu_{n}\right), \phi(\varsigma)\right)
\end{aligned}
$$

for all $n \in \mathbb{N}$.

Finally, letting $n \rightarrow+\infty$ in the above calculations and using that $H$ is continuous in $(0,0,0)$, we deduce that $d(\varsigma$, $Y \varsigma) \leq H(0,0,0)=0$; that is, $\varsigma=Y \varsigma$.

Example 15. Let $M=[0,1]$ endowed with the standart metric $d(\mu, \gamma)=|\mu-\gamma|$ for all $\mu, \gamma \in M$. Consider the mapping $Y$ $: M \rightarrow M$ defined by

$$
Y \mu=\left\{\begin{array}{ll}
\mu / 2 ; & \mu \in 0,1) \\
1 ; & \mu=1
\end{array} .\right.
$$

Clearly, $Y$ is not a $F$ - contraction but $Y$ is an almost $F-H$ -contraction with respect to the functions $F \in \mathscr{F}$ defined by $F(\alpha)=\ln \alpha$ for all $\alpha>0, H \in \mathscr{H}$ defined by $H(a, b, c)=\max$ $\{a, b\}+c$ for all $a, b, c \in 0,+\infty$, the real number $\tau=\ln 2$ and $L=4$, and a l.s.c. function $\phi: M \rightarrow 0,+\infty, \phi(t)=t$ for all $t \in M$, indeed.

Case 1. $\mu=0, \gamma=1$, we have

$$
\begin{aligned}
\tau+ & F(H(d(Y \mu, Y \gamma), \phi(Y \mu), \phi(Y \gamma))) \\
& =\tau+F(H(d(Y 0, Y 1), \phi(Y 0), \phi(Y 1))) \\
& =\tau+F(H(d(0,1), \phi(0), \phi(1))) \\
& =\tau+F(H(1,0,1))=\ln 4 \\
& \leq \ln 6=F(H(d(0,1)+4 d(1, Y 0), \phi(0), \phi(1))) \\
& =F(H(d(\mu, \gamma)+L d(\gamma, Y \mu), \phi(\mu), \phi(\gamma))) .
\end{aligned}
$$

Case 2. $\mu=1, \gamma=0$, we have

$$
\begin{aligned}
\tau+ & F(H(d(Y \mu, Y \gamma), \phi(Y \mu), \phi(Y \gamma))) \\
& =\tau+F(H(d(Y 1, Y 0), \phi(Y 1), \phi(Y 0))) \\
& =\tau+F(H(d(1,0), \phi(1), \phi(0))) \\
& =\tau+F(H(1,1,0))=\ln 2 \leq \ln 5 \\
& =F(H(d(1,0)+4 d(0, Y 1), \phi(1), \phi(0))) \\
& =F(H(d(\mu, \gamma)+L d(\gamma, Y \mu), \phi(\mu), \phi(\gamma))) .
\end{aligned}
$$

Case 3. $\mu, \gamma \in(0,1)$ with $\mu>\gamma$, we have

$$
\begin{aligned}
\tau+ & F(H(d(Y \mu, Y \gamma), \phi(Y \mu), \phi(Y \gamma))) \\
& =\tau+F\left(H\left(d\left(\frac{\mu}{2}, \frac{\gamma}{2}\right), \phi\left(\frac{\mu}{2}\right), \phi\left(\frac{\gamma}{2}\right)\right)\right) \\
& =\tau+F\left(H\left(\frac{\mu-\gamma}{2}, \frac{\mu}{2}, \frac{\gamma}{2}\right)\right) \\
& =\tau+F\left(\frac{\mu+\gamma}{2}\right)=\ln (\mu+\gamma) \\
& \leq \max \{\ln (\mu+\gamma), \ln (4 \gamma-\mu)\} \\
& =F(H(d(\mu, \gamma)+L d(\gamma, Y \mu), \phi(\mu), \phi(\gamma))) .
\end{aligned}
$$

\section{Data Availability}

No data were used to support this study.

\section{Conflicts of Interest}

The authors declare that they have no conflicts of interest.

\section{References}

[1] S. Banach, "Sur les opérations dans les ensembles abstraits et leur application aux équations intégrales," Fundamenta Mathematicae, vol. 3, pp. 133-181, 1922.

[2] M. Abbas, M. Berzig, T. Nazir, and E. Karapinar, "Iterative approximation of fixed points for presic type F-contraction operators," University Politehnica Of Bucharest Scientific Bulletin-Series A-Applied Mathematics And Physics, vol. 78, no. 2, pp. 147-160, 2016.

[3] E. Karapinar, P. Kumam, and P. Salimi, "On $\alpha$ - $\psi$-Meir-Keeler contractive mappings," Fixed Point Theory and Applications, vol. 2013, no. 1, 2013.

[4] E. Karapinar, A. Fulga, and R. P. Agarwal, "A survey: Fcontractions with related fixed point results," Journal of Fixed Point Theory and Applications, vol. 22, no. 3, p. 69, 2020.

[5] E. Karapinar, M. Kutbi, H. Piri, and D. O'Regan, "Fixed points of conditionally F-contractions in complete metric-like spaces," Fixed Point Theory and Applications, vol. 2015, no. 1, 2015.

[6] Ö. Acar, "Rational type multivalued F G -Contractive mappings with a graph," Results in Mathematics, vol. 73, no. 2, p. 52, 2018.

[7] I. A. Rus, A. Petrusel, and G. Petrusel, Fixed Point Theory, Cluj University Press, Cluj-Napoca, 2008.

[8] D. Wardowski, "Fixed points of a new type of contractive mappings in complete metric spaces," Fixed Point Theory and Applications, vol. 2012, no. 1, 2012.

[9] M. Jleli, B. Samet, and C. Vetro, "Fixed point theory in partial metric spaces via $\varphi$-fixed point's concept in metric spaces," Journal of Inequalities and Applications, vol. 2014, no. 1, 2014.

[10] H. H. Alsulami, E. Karapinar, and H. Piri, "Fixed points of modified -contractive mappings in complete metric-like spaces," Journal of Function Spaces, vol. 2015, Article ID 270971, 9 pages, 2015.

[11] H. Aydi, E. Karapinar, and H. Yazidi, "Modified Fcontractions via $\alpha$-admissible mappings and application to integral equations," Filomat, vol. 31, no. 5, pp. 1141-1148, 2017. 
[12] E. Karapinar, H. Piri, and H. H. AlSulami, "Fixed points of generalized F-Suzuki type contraction in complete b-metric spaces," Discrete Dynamics in Nature and Society, vol. 2015, Article ID 969726, 8 pages, 2015.

[13] C. Vetro, "A fixed-point problem with mixed-type contractive condition," Constructive Mathematical Analysis, vol. 3, no. 1, pp. $45-52,2020$. 